\title{
HEMOS-Cloud: HPC Cloud-based Modeling and Simulation Platform for HEMOS Software
}

\author{
Myungil Kim ${ }^{1}$, Dongwoo Seo ${ }^{1}$, Daeyong Jung ${ }^{1}$ and Jaesung Kim ${ }^{1}$ \\ ${ }^{1}$ Korea Institute of Science and Technology Information \\ 245 Daehak-ro, Yuseong-gu, Daejoen, 34141, Korea \\ \{mikim, seodongwoo, daeyongjung, jaesungkim\}@kisti.re.kr
}

\begin{abstract}
Modeling \& Simulation $(M \& S)$ is a technology that replaces the physical production and experimentation of products with virtual production (modeling) and engineering analysis (simulation). $M \& S$ can greatly reduce the time and cost it takes to develop new products and thus increasing the manufacturing company's competitiveness. This paper describes the construction of M\&S platform based on High Performance Computer (HPC) cloud. High performance Engineering MOdeling \& Simulation (HEMOS) is a $M \& S$ software developed to support structural analysis and fluid analysis. Developed using open-source software, it supports all $M \& S$ processes from pre-processing to analysis and post-processing. HEMOS is developed to simplify shapes, create mesh, and detect contact surfaces at the pre-processing. It uses CalculiX and OpenFOAM, both open-source solvers, to analysis structural and fluid problems without license restrictions at the analysis while OpenGL and Paraview were used to implement post-processing. HEMOS is provided through HEMOS-Cloud which was built based on Xen server with 532 CPU cores and is connected with KISTI's supercomputer of about 300TF. This HPC Cloud-based modeling and simulation platform that services HEMOS can increase user accessibility and reduce costs.
\end{abstract}

Keywords: Modeling, Simulation, Cloud Computing, High Performance Computing

\section{Introduction}

Digital manufacturing (DM) using high performance computer (HPC) and M\&S have been becoming the key tools that can strengthen national manufacturing competitiveness around the world [1][2]. Major developed countries emphasize convergence of information and communication technology (ICT) and manufacturing, and promote various manufacturing innovation policies related to this. The most representative manufacturing innovation policies include A National Strategic Plan for Advanced Manufacturing (USA, 2012), Industrie 4.0 (Germany, 2012), and Japan Revitalization Strategy (Japan, 2013). In particular, M\&S is attracting attention as a technology that can replace physical prototyping and experimentation with virtual product creation (modeling) and engineering analysis (simulation), which dramatically shortens the time and cost of product design and verification. For example, Boeing manufactured and tested 77 wings to design Boeing-767 in 1980's but when designing the Boeing-787 or 747-8 utilizing M\&S technology, Boeing only manufactured 7 wings for testing. Boeing was able to save significant costs for wind tunnel testing through this [3]. Goodyear cooperated with Sandia National Laboratories in New Mexico to develop and apply a product development process utilizing $M \& S$ and reduced its key product design time from 3 years to within a year. Also, the expenditures on tire building and testing dropped from 40 percent of the company's research, design engineering and quality (RDE\&Q) budget to 15 percent [4]. 
While the need and importance of M\&S is increasingly growing, many small and medium-sized manufacturing enterprises (SME) are struggling to utilize M\&S due to lack of computing resources, professional manpower, and high M\&S software prices. To solve these problems, we designed and developed high performance engineering modeling \& simulation (HEMOS) and HEMOS-Cloud, a HPC cloud-based M\&S service platform. HEMOS is M\&S software developed to support structural analysis and fluid analysis and uses open-source solvers such as CalculiX and OpenFOAM to solve highly expensive license costs. HEMOS supports all $M \& S$ processes from pre-processing to analysis and post-processing, and provides a workflow-based, easy and convenient user interface. The software also handles complex processes such as geometry simplification, mesh generation, and contact surface detection. HEMOS-Cloud is a platform to service HEMOS and was built with a pay-per-use cloud to solve expensive computing resource problem. Section 2 of this paper introduces relevant M\&S software and Cloud-based M\&S services. In Section 3 and 4, we discuss the details of the design and respective results of HEMOS and HEMOS-Cloud implementations. Finally, Section 5 describes the conclusion and directions for future research.

\section{Related Works}

Generally, M\&S refers to using models - physical, mathematical, or other forms of logical representation of a system, entity, phenomenon, or process - as a basis for simulations - methods for implementing a model (either statically or) over time - to develop data as a basis for managerial or technical decision making. In case of manufacturing $M \& S$, model and simulation refer to the CAD file and engineering analysis such as structural and fluid analysis respectively. The M\&S applied in this paper is manufacturing $\mathrm{M} \& S$ which consists of pre-processing, analysis and post-processing as shown in Table 1.

Table 1. M\&S Processes

\begin{tabular}{|c|l|}
\hline Process & \multicolumn{1}{c|}{ Key features } \\
\hline Pre-processing & $\begin{array}{l}\text { Creation/ simplification of a product's shape, mesh generation } \\
\text { for analysis, setting of material and load, and setting of boundary } \\
\text { conditions }\end{array}$ \\
\hline Solving & $\begin{array}{l}\text { Perform numerical analysis using solvers and HPC according to } \\
\text { analysis fields (Structure, CFD, Dynamics, Molding, etc.) }\end{array}$ \\
\hline Post-processing & Visualization of analysis results \\
\hline
\end{tabular}

The most common open-source M\&S software are Code Aster, Elmer, CalculiX, and Salome as shown below in Figure 1. Code Aster is finite-element package software that was released in 2001 as an open-source type based on GNU GPL developed by EDF in France and it is mostly composed of Fortran and Phyton programming language. Code Aster is used mostly for dynamics analysis based on finite-element method and it is also capable of analyzing linear/non-linear statics and dynamics along with fatigue, damage, fracture, contact, geo-materials, porous media, and others [5]. Elmer is open-source multiphysics analysis software developed by CSC, a partner institute to Finnish universities, research institutes, and industries in Finland since 1995 and it supports structure dynamics, hydrokinetics, electromagnetic field, heat transfer, and noise analysis [6]. CalculiX was developed by Guido Dhondt of Germany since 1998 and it has a nearly identical input file as ABAQUS, commercial software. CalculiX supports linear static analysis, non-linear static analysis, modal analysis, buckling analysis, heat transfer analysis, and other various forms of analysis and it is even capable of analyzing elastic, elasto-plastic, hyper-elastic, and some visco-elastic materials [7]. SALOME is open-source software that provides a generic platform for Pre- and Post-Processing for numerical simulation. It is based on an 
open and flexible architecture made of reusable components. SALOME is a crossplatform solution and is distributed as open-source software under the terms of the GNU LGPL license [8]. However, such open-source M\&S software does not support pre/postprocessors which makes it incapable of supporting all M\&S processes along with complications in its usage method and lack of variety in terms of its range of analysis.

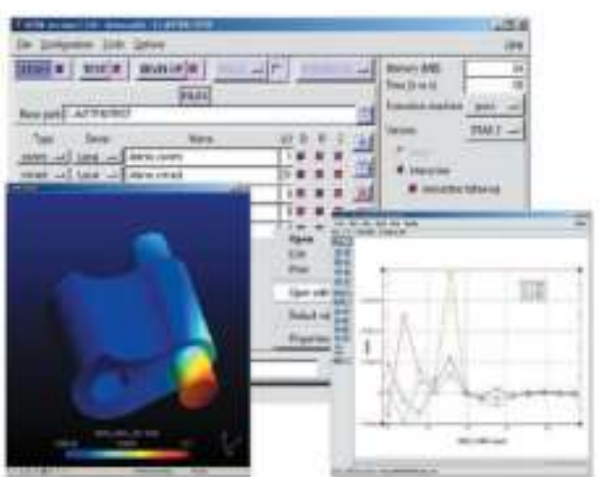

(a) Code_Aster

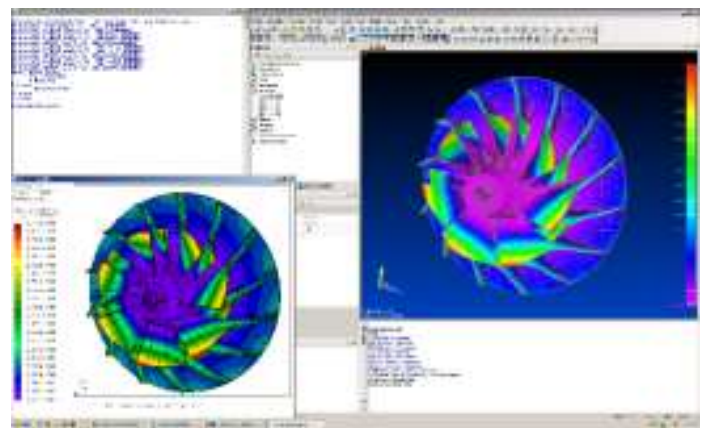

(c) Calculix

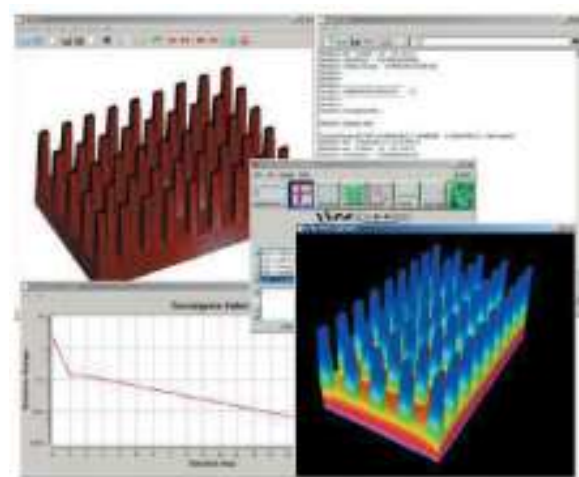

(b) Elmer

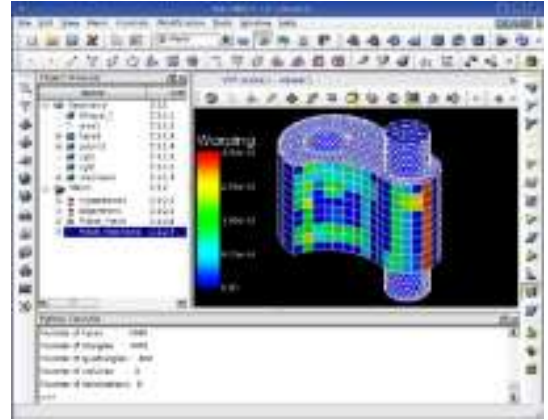

(d) Salome

Figure 1. Open-Source M\&S Software

Cloud-based service can make any resources such as software, platform and infrastructure available to users on demand via the Internet from a cloud computing provider's server. Recently, M\&S software is being transformed into a cloud-based service instead of a stand-alone service. Converting M\&S software to cloud-based services can improve accessibility, reduce costs, and make better use of computing resources. As representative commercial cloud-based M\&S services, there are Simscale, ESI cloud, Fortissimo, Rescale, etc., as shown in Figure 2. Simscale is a cloud-based CAE platform that lets users seamlessly simulate, share and collaborate in the fields of fluid dynamics (CFD), finite element analysis (FEA) and thermodynamics [9]. ESI cloud offers designers and engineer cloud-based $M \& S$ solutions across physics and engineering disciplines with browser based modeling, visualization and real-time collaboration tools [10]. Fortissimo is a collaborative project that enables European SMEs to be more competitive globally through the use of simulation services running on a High Performance Computing cloud infrastructure [11]. Fortissimo provides one-stop, pay-peruse, on-demand access to advanced simulation and modeling resources including software, hardware and expertise. Rescale is a cloud simulation platform that helps engineers and scientists build, compute, analyze, and scale simulations with high performance computing resources [12]. 


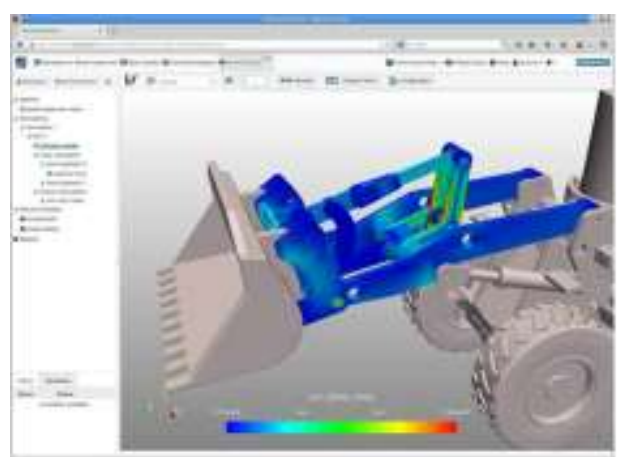

(a) Simscale

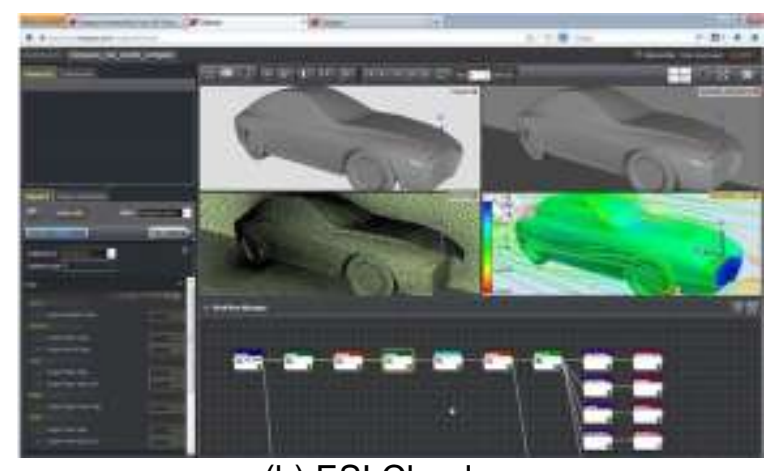

(b) ESI Cloud

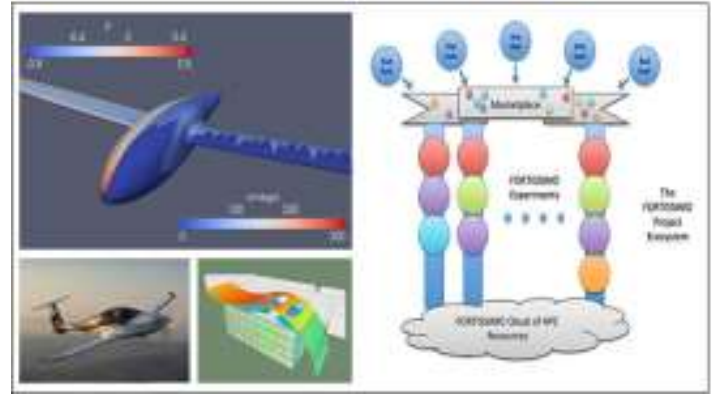

(c) Fortissimo

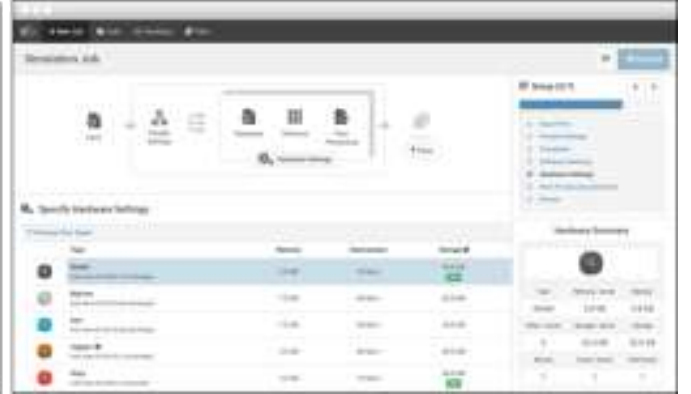

(d) Rescale

Figure 2. Cloud-based M\&S Services

\section{Design and Implementation of HEMOS}

As Table 2 shows, HEMOS is composed of customized M\&S software that predicts the performance of widely used M\&S SW that supports structure analysis and fluid analysis, tube insertion/separation force, bracket, HVAC, and Bending. Developed using opensource software, HEMOS supports all M\&S processes including pre-processing, analysis and post-processing.

Table 2. HEMOS SW Suite

\begin{tabular}{|c|c|l|c|}
\hline \multicolumn{2}{|c|}{ Category } & \multicolumn{1}{|c|}{ Characteristics } & Solver/Tool \\
\hline $\begin{array}{c}\text { General-Purposed } \\
\text { M\&S SW } \\
\text { (HEMOS) }\end{array}$ & Structure & $\begin{array}{l}\text { Linear Static, Non-linear Contact } \\
\text { Analysis }\end{array}$ & CalculiX \\
\cline { 2 - 4 } & Fluid & $\begin{array}{l}\text { Steady state/Incompressible } \\
\text { Turbulence Analysis }\end{array}$ & OpenFOAM \\
\cline { 2 - 4 } & Tube & $\begin{array}{l}\text { Tube-Clip Insertion/Separation } \\
\text { Analysis }\end{array}$ & CalculiX \\
\cline { 2 - 4 } $\begin{array}{c}\text { Customized } \\
\text { M\&S SW } \\
\text { (HEMOS+) }\end{array}$ & Bracket & $\begin{array}{l}\text { Refrigerator Bracket Stress-Strain } \\
\text { Analysis }\end{array}$ & CalculiX \\
\cline { 2 - 4 } & HVAC & $\begin{array}{l}\text { Web-based Air Cleaner Performance } \\
\text { Analysis }\end{array}$ & OpenFOAM \\
\cline { 2 - 4 } & Bending & $\begin{array}{l}\text { Spring Back Estimation According to } \\
\text { Tube Bending Utilizing Optimization } \\
\text { Method }\end{array}$ & OptisLang \\
\hline
\end{tabular}




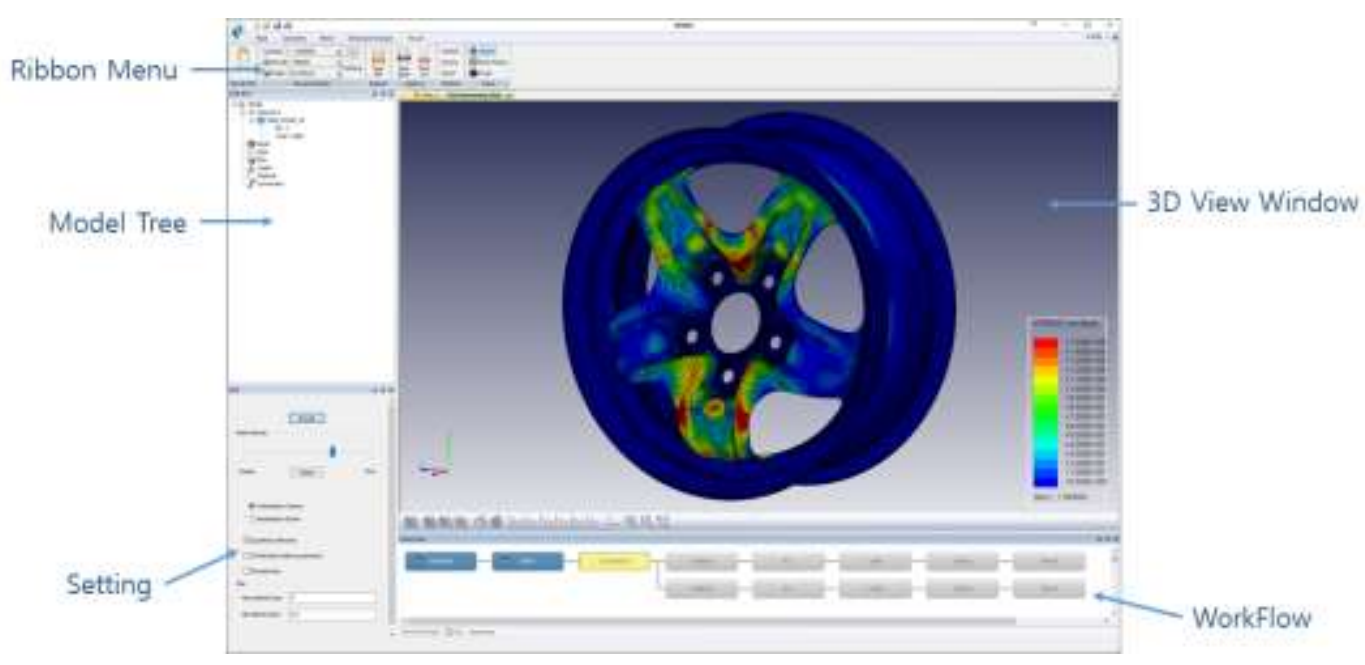

Figure 3. Integrated User Interface of HEMOS

\subsection{HEMOS-Structure and Fluid}

Basically, HEMOS is an entry level M\&S software developed to support structural analysis and fluid analysis. HEMOS provides workflow-based step-by-step analysis processes and wizard feature to help product designers with inadequate knowledge of engineering analysis that predicts performances of designed products easily. In other words, the software provides predefined workflows according to the analysis field and product groups that the user selected through the wizard feature. Also, it maximizes user convenience by providing an integrated user interface tightly linked with the ribbon menu, model tree, setup window, 3D view window, and workflow as shown in Figure 3.

Generally, an open-source solver is advantageous in that there is no restriction on the number of computing nodes or CPU/GPU cores, yet there is a drawback that there are few pre-processors with excellent usability. To address this issue, HEMOS provides a preprocessor that automates complex functions such as shape simplification, mesh generation, and contact surface detection based on Open CasCade, an open-source 3D CAD kernel. Mesh generation automatically generates mesh based on the physical size, curved surfaces, and element type of the model using the suite of meshing software components MeshGems.
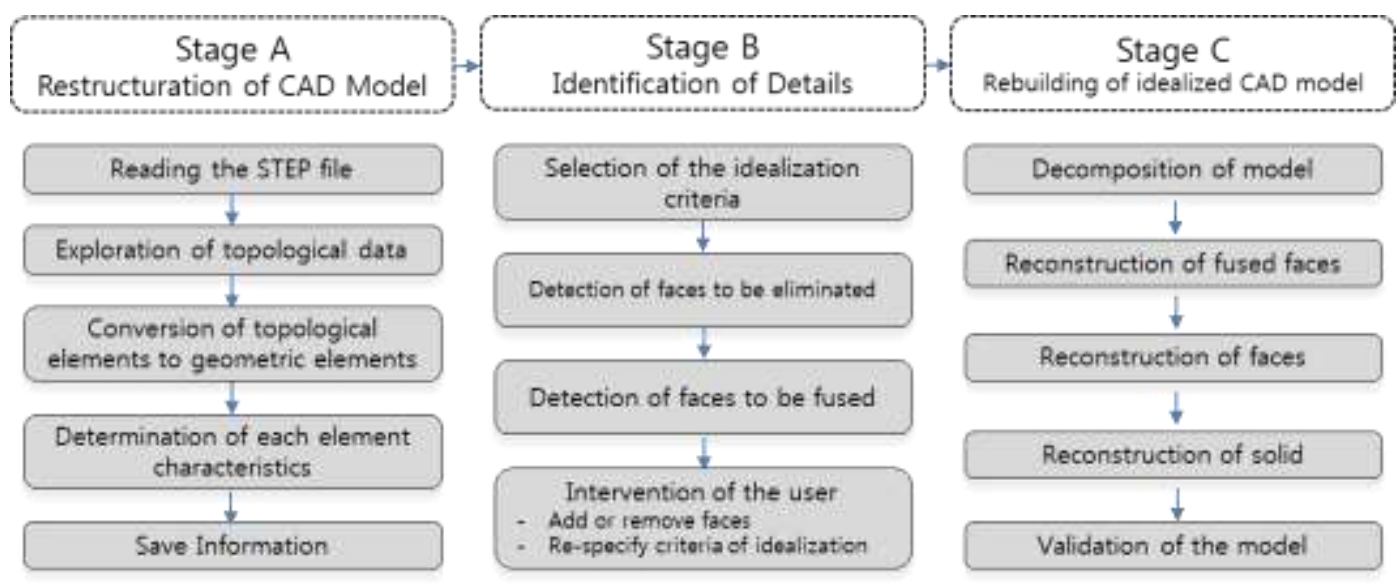

Figure 4. Automatic Geometry Simplification Processes 
Automatic geometry simplification provides the ability to find and remove small edges, faces, holes, engraved characters, and embossed characters according to user-specified constraints. Geometry simplification is performed through a 3-step procedure of restructuration of CAD model, identification of details, and rebuilding of idealized CAD model and the details of each step are shown below in Figure 4. Contact surface detection automatically searches contact surfaces between parts through grouping and parallelized calculation according to procedures as shown in Figure 5. After deducing the list of solid models from the entire assembly model, single solid models are generated based on the list and the group of candidates that may come into contact with one another. Proximity parallel processing based on bounding box is performed on this group of candidates and the surface factors are separated and a group of candidates for contact surface is generated. Lastly, the surface parameters such as isocurve, normal vector, surface on surface are calculated to determine contact surface.

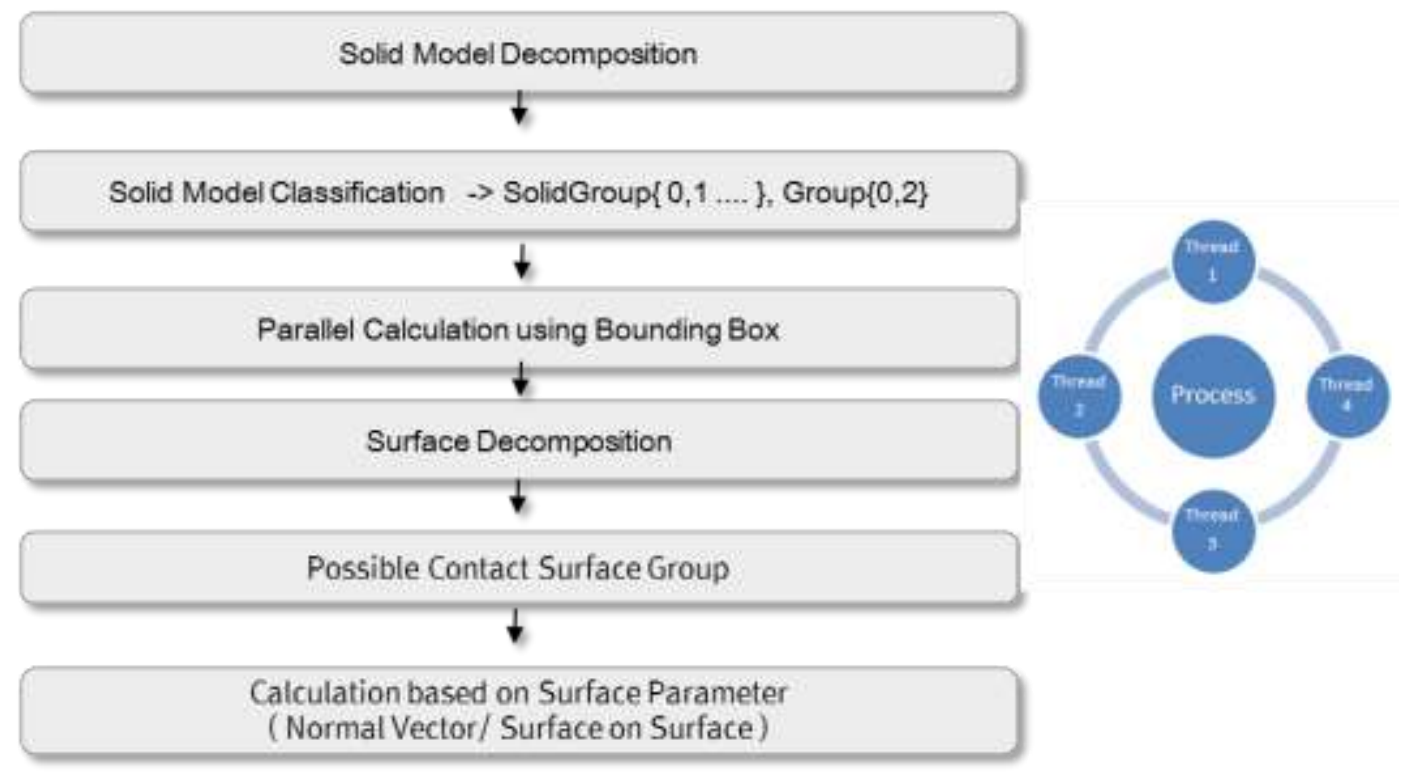

Figure 5. Parallelized Contact Surface Detection Processes

For structural analysis, it uses CalculiX, an open-source solver, and our own HEMOSFEM solver; for fluid analysis the open-source solver OpenFOAM is used. It uses conditions that were set during the pre-processing to automatically create input files for the solver and the files are automatically transferred to the HEMOS's analysis resources or to the supercomputer. Once the analysis is completed, the structural analysis results are 3D visualized using a post-processor developed based on OpenGL; fluid analysis results are 3D visualized using the open-source visualization application Paraview.

\subsection{HEMOS+: Customized M\&S Software}

HEMOS+ is customized M\&S software for simulations of specific products or processes and it was developed using the modules developed for pre-processing, solving, and post-processing of HEMOS-Structure and HEMOS-Fluid. Currently, HEMOS+ is composed of 4 software in the form of tube, bracket, HVAC, and bending as shown in Figure 6. They provide a predefined workflow for each target product and technology, and the user can proceed with the analysis sequentially according to the given workflow. 


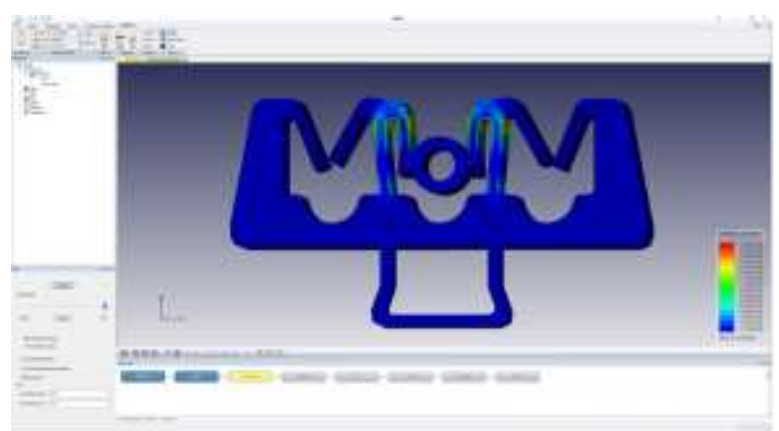

(a) HEMOS-Tube



(c) HEMOS-BRK

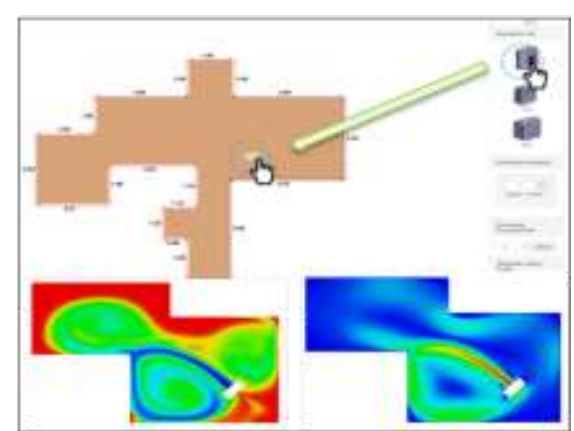

(b) HEMOS-HVAC

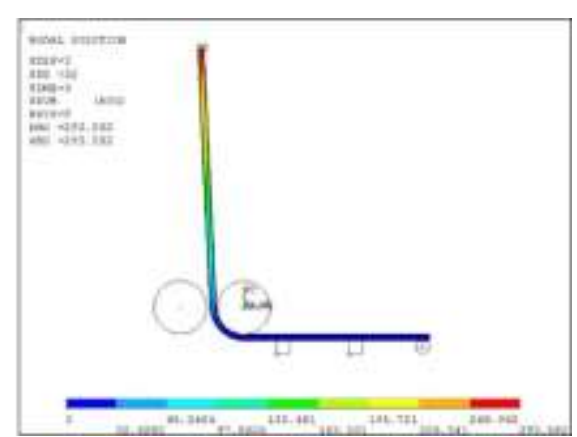

(d) HEMOS-Bending

Figure 6. HEMOS+ for Tube, HVAC, Bracket and Bending

HEMOS-Tube is M\&S software that predicts the insertion force and separation force between tubes for fuel and break oil and clips in automobiles and its process is composed of automatic generation of tube shape, automated search of contact surface, automatic generation of mesh, automated configuration of parameters for solving, and automatic post-processing of analysis results. HEMOS-BRK is M\&S software for analysis the stress and strain of brackets for refrigerators and it provides a simple workflow through a simple 3 -step process so the designer can predict the stress and strain of the brackets conveniently. HEMOS-BRK features functions such as automatic generation of bracket shape through entry of parameters on bracket components such as bracket body and bead, automatic generation of mesh in consideration of analysis accuracy, automatic generation of load and boundary conditions, and automatic generation of report including the results on stress and strain post-processing. HEMOS-HVAC is M\&S software that predicts the performance of the air cleaning system and it was developed with a web-based (HTML5) user interface. It features functions such as air cleaning target space selection, air cleaner model selection and placement, and wind speed and running time configuration and the pimpleSpeciesFoam is used to perform fluid analysis. Also, it was developed to process multiple user requests more efficiently utilizing the Son of Grid Engine (SGE) based batch scheduler. HEMOS-Bending is 1-D solver based M\&S software to predict the spring back value based on tube's bending. Parameter values such as the bending angle, tube diameter, and tube material are altered as the simulations are performed and the results saved for the database. Optimization method is applied based on the constructed database and a meta-model that predicts the spring back value is derived and the user can predict spring back values without simulations by just entering design parameters. 




Figure 7. Conceptual Diagram of HEMOS-Cloud

\section{HEMOS-Cloud}

HEMOS-Cloud is an HPC cloud-based platform for servicing HEMOS software including HEMOS-Structure, HEMOS-Fluid and HEMS+ as shown in Figure 7. Also, HEMOS-Cloud supports other software besides HEMOS such as Salome, Paraview, and DAFUL. HEMOS-Cloud provides users with Windows environment and HEMOS software based on virtual desktop infrastructure (VDI) using XenServer, an open-source virtualization platform. The HEMOS-Cloud is built with $532 \mathrm{CPU}$ cores, 11,520 CUDA GPU cores, and storage of 57.5 TB and consists of four hardware groups: master, analysis, design and development, for effective resource utilization and management. The detailed specifications for each group are shown in Table 3. Master plays the role of asset distribution manager as the user requests are assigned to calculation resources and checks the amount of work being processed to assign new workload. The analysis group does not support pre-processing or post-processing but it receives the job script generated by the user and performs the solving process and the design group supports pre/post-processing including CAD modeling and mesh generation. The development group provides a Linux environment and supports sequential code parallelization and development using Linuxonly application.

Table 3. HEMOS-Cloud Specifications

\begin{tabular}{|c|c|l|c|c|}
\hline Group & EA & \multicolumn{1}{|c|}{ Features } & $\begin{array}{c}\text { CPU } \\
\text { cores }\end{array}$ & OS \\
\hline Master & 1 & $\begin{array}{l}\text { - User and task management } \\
\text { - Web-based user environment }\end{array}$ & 16 & $\begin{array}{c}\text { RedHat Linux } \\
6.7\end{array}$ \\
\cline { 2 - 5 } & 1 & $\begin{array}{l}\text { - File server } \\
\text { - User accounts and directories }\end{array}$ & 12 & Windows 2012 \\
\hline Analysis & 9 & - Numerical analysis resources & 192 & $\begin{array}{c}\text { RedHat Linux } \\
6.7\end{array}$ \\
\hline Design & 7 & - Windows desktop environment & 224 & $\begin{array}{c}\text { XenServer 6.2 } \\
\text { (Windows 7) }\end{array}$ \\
\hline Development & 5 & $\begin{array}{l}\text { - Linux desktop environment } \\
\text { - Linux development environment }\end{array}$ & 88 & $\begin{array}{c}\text { RedHat Linux } \\
6.7\end{array}$ \\
\hline
\end{tabular}


The basic resource management and monitoring functions are provided to manage job, connection, user and process. HEMOS-Cloud provides a job scheduling function capable of dynamic allocation and load balancing of user operations using SGE (Son of Grid Engine), an open-source distributed resource management system. HEMOS-Cloud works with KISTI's supercomputer TACHYON2 through agent-to-agent communication as shown in Figure 8. HEMOS software users with a Tachyon2 account can $\log$ in to Tachyon2 via OTP (One Time Password) method, and can perform HEMOS engineering analysis, upload and download data, and monitor jobs.

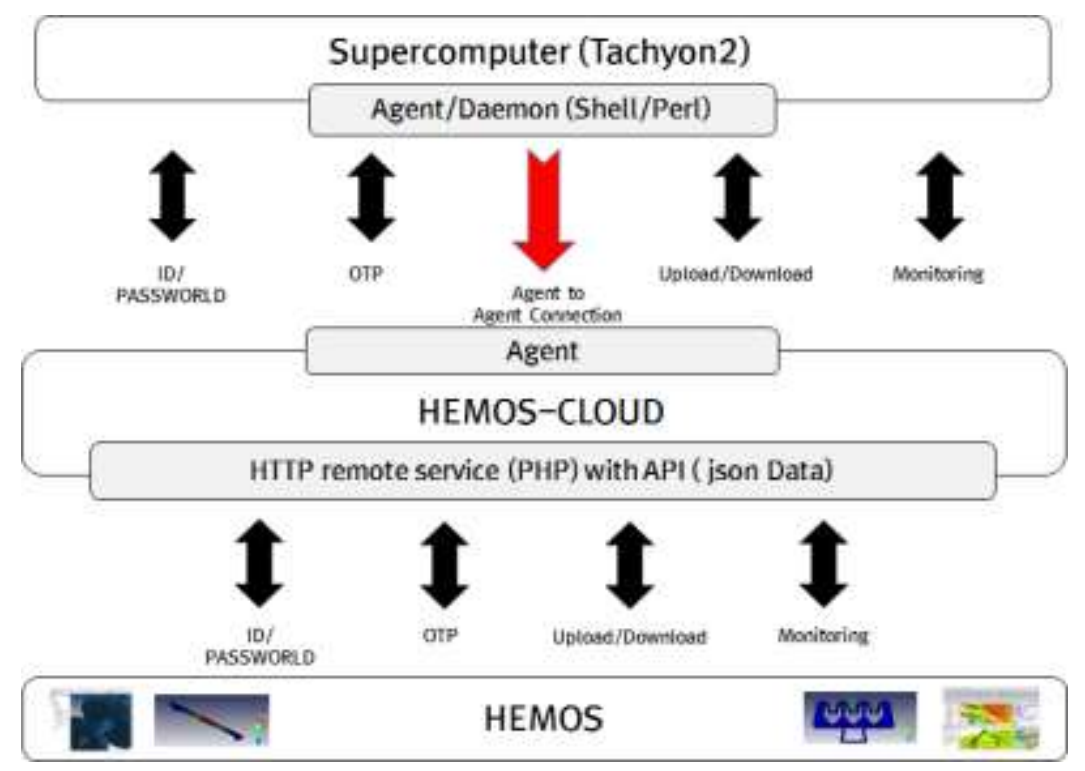

Figure 8. Communication of HEMOS and Supercomputer (Tachyon2)

\section{Conclusion}

In this paper, we describe HEMOS, entry-level M\&S software developed for designers and engineers of manufacturing SMEs, and HEMOS-Cloud, a service platform based on HPC cloud. HEMOS consists of two general purpose M\&S software and four customized M\&S software developed to support all M\&S processes such as pre-processing, analysis and post-processing. The general purpose $M \& S$ software supports structural and fluid analysis whilst customized M\&S software specialize in air cleaning system, bracket, tube, and bending. Particularly, we developed and applied an integrated UI using a workflowbased step-by-step method for easy and convenient use, and developed a pre-processor that automates complex functions such as geometry simplification, mesh generation, and contact surface detection. Also, we used open-source libraries and solvers such as Open CasCade, CalculiX, OpenFOAM, and Paraveiw to develop HEMOS software to distribute licenses without restrictions. HEMOS-Cloud consists of four hardware groups such as master, analysis, design and development for effective resource utilization and management as an HPC cloud-based M\&S service platform. HEMOS-Cloud provides job scheduling function using SGE and is developed to enable large-scale analysis by communicating with KISTI's supercomputer Tachyon2. In the future, we will improve the automation performances of pre-processing functions such as shape healing and mesh generation, and expand the field of HEMOS' general-purpose analysis. Moreover, we will provide users with safe and efficient services by stabilizing HEMOS-Cloud, expanding resources, and strengthening securities. 


\title{
References
}

[1] Tuncer I. Oren, "The Importance of a Comprehensive and Integrative View of Modeling and Simulation", Proceedings of the 2007 Summer Computer Simulation, San Diego, USA, (2007) July 1518.

[2] Xiaoning Jin, David Siegel, Brian A. Weiss, Ellen Gamel, Wei Wang, Jay Lee and Jun Ni, "The Present Status and Future Growth of Maintenance in US Manufacturing: Results from a Pilot Survey", Manufacturing Review, vol. 3, (2016).

[3] Boeing Catches a Lift with High Performance Computing. U.S. Council of Competitiveness publication 2009.

[4] Goodyear Puts the Rubber to the Road with High Performance Computing, U.S. Council of Competitiveness publication 2009.

[5] Code_Aster: Structures and Thermomechanics Analysis for Studies and Research , http://www.codeaster.org

[6] Elmer: An Open Source Multiphysical Simulation Software, https://www.csc.fi/web/elmer

[7] CalculiX: A free Software Three-Dimensional Structural Finite Element Program, http://www.calculix.de

[8] Salome: The Open-Source Integration Platform for Numerical Simulation, http://www.salomeplatform.org

[9] Daniel Zehe, Wentong Cai, Alois Knoll and Hiko Aydt, "Tutorial on a Modeling and Simulation Cloud Service", Proceedings of the 2015 Winter Simulation Conference, Huntington Beach, USA, (2015) December 6-9.

[10] CAE Solution on the Cloud - ESI Group, http://cloud.esi-group.com

[11] Wolfgang Ziegler, Roberto Dippolito, Massimo Dauria, Jochem Berends, Maarten Nelissen and Ramon Diaz, "Implementation a "one-stop-shop" Providing SMEs with Integrated HPC Simulation Resources using Fortissimo Resources", Proceedings of eChallenges e-2014, Belfast, Ireland, (2014), October 2930.

[12] Rescale, Cloud HPC Simulation Platform, http://www.rescale.com

\section{Acknowledgement}

This Paper is a revised and expanded version of a paper entitled "Cloud-based Modeling and Simulation Platform" presented at GST2016, Jeju, Korea, and December 21-23, 2016.

\begin{abstract}
Authors
Myungil Kim, he is a senior researcher at Korea Institute of Science and Technology Information (KISTI), Korea. He received his BS, MS and Ph.D. degrees in Computer Science and Engineering from Chungang University, Korea, in 1998, 2000, and 2004, respectively. His current research interests include modeling and simulation, cloud computing, supercomputing and research network.
\end{abstract}
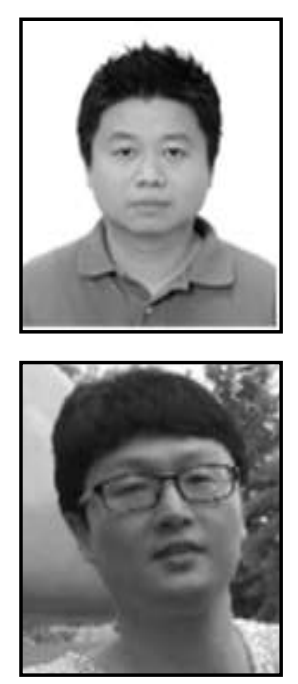

Dongwoo Seo, he is a senior researcher at Korea Institute of Science and Technology Information (KISTI), Korea. He received his BS, MS and Ph.D. degrees in Industrial Engineering from Chonnam National University, Korea, in 2003, 2008, and 2012, respectively. His current research interests include AR/VR applications, software architecture, user experience (UX) in CAE service and collaborative design. 


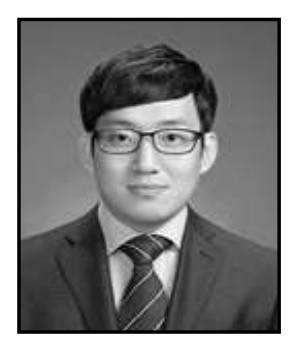

Daeyong Jung, he is a senior researcher at Korea Institute of Science and Technology Information (KISTI), Korea. He received Integrated Master and Ph.D. degrees in the Department of Computer Science Education of Korea University, Seoul, Korea, in 2015. His main research interests are cloud computing, grid computing, distributed computing, and fault-tolerance systems.

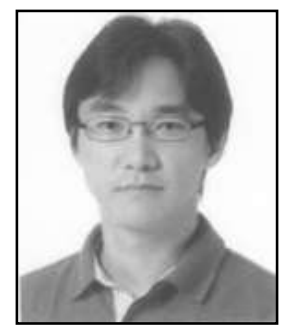

Jaesung Kim, he is a principal researcher at Korea Institute of Science and Technology Information (KISTI), Korea. He received his $\mathrm{MS}$ and $\mathrm{Ph} . \mathrm{D}$. degrees in Industrial Engineering from Pohang University of Science and Technology (POSTECH), Korea, in 1999, and 2003, respectively. His current research interests include product design, large-scale simulation, VR/AR based post-processing. 
International Journal of $\mathrm{u}-$ and $\mathrm{e}-$ Service, Science and Technology Vol.10, No.8 (2017) 